\title{
Effects of nonlinear dispersion on squeezed states in two-photon devices
}

\author{
P. García-Fernández and F. J. Bermejo \\ Instituto de Estructura de la Materia del Consejo Superior de Investigaciones Científicas, Serrano 119, \\ E-28006 Madrid, Spain \\ (Received 1 July 1988)
}

\begin{abstract}
The deleterious effects of nonlinear dispersion on squeezed light in two-photon devices when absorption losses are included have been analyzed making use of the variables called two-mode quadrature-phase amplitudes. The uncertainties in the quadrature amplitudes have been computed from a generalized Fokker-Planck equation. The dependence of squeezing on the nonlinear coupling, the modulation (including the case of high modulations), the pumping phase (where nonlinear dispersion causes an intensity-dependent shift in the minimum squeezing), and the absorption losses have been studied.
\end{abstract}

\section{INTRODUCTION}

Levenson et al. ${ }^{1}$ have experimentally demonstrated the importance of nonlinear dispersion in the squeezing process in parametric interactions. They have pointed out ${ }^{2}$ that the nonlinear susceptibility also causes an intensitydependent phase shift between the pump and the signal and idler modes in two-photon devices. This effect can be taken into account by means of an additional term in the interaction Hamiltonian. Milburn et al ${ }^{3}$ have presented a unified treatment of two-mode squeezed-state generation in optical fibers. In this article we study the influence of nonlinear dispersion on the uncertainties of the two-mode quadrature-phase amplitudes by means of new variables introduced by Caves and Schumaker. ${ }^{4}$

An intense laser beam at frequency $2 \Omega$-the pump beam - that illuminates a suitable nonlinear medium will be considered. The output light consists of pairs of simultaneously emitted photons which excite pairs of modes at frequencies $\Omega \pm \epsilon$, where $\epsilon<\Omega$ is a modulation frequency. The annihilation operators for the two modes are denoted by $a_{+}$and $a_{-}$, and satisfy the usual commutation relations

$$
\begin{aligned}
& {\left[a_{+}, a_{-}\right]=\left[a_{+}, a_{-}^{\dagger}\right]=0,} \\
& {\left[a_{+}, a_{+}^{\dagger}\right]=\left[a_{-}, a_{-}^{\dagger}\right]=1 .}
\end{aligned}
$$

The free Hamiltonian for the two modes is given by

$$
\begin{aligned}
& H_{0}=\hbar(\Omega+\epsilon) a_{+}^{+} a_{+}+\hbar(\Omega-\epsilon) a_{-}^{+} a_{-}=H_{R}+H_{M}, \\
& H_{R}=\hbar \Omega\left(a_{+}^{\dagger} a_{+}+a_{-}^{\dagger} a_{-}\right), \\
& H_{M}=\hbar \epsilon\left(a_{+}^{\dagger} a_{+}-a_{-}^{\dagger} a_{-}\right) .
\end{aligned}
$$

The Hamiltonian for an ideal two-photon process in the Schrödinger picture (SP) can be written

$H=H_{0}+\frac{\hbar}{2} i \kappa(t)\left(a_{+} a_{-} e^{-2 i(\varphi-\Omega t)}-a_{+}^{\dagger} a_{-}^{\dagger} e^{2 i(\varphi-\Omega t)}\right)$,

where $\kappa(t)$ is an arbitrary real function of time. The process is characterized by the function $\kappa(t) e^{2 i(\varphi-\Omega t)}$, and may be used to describe, for example, an ideal parametric amplifier.

We will take into account the effects of nonlinear dispersion by means of the Hamiltonian:

$$
H_{D}=\hbar \kappa^{\prime}\left(a_{+}^{+} a_{+}+a_{-}^{\dagger} a_{-}\right),
$$

which represents a pump-induced phase shift of the side bands.

The following variables, ${ }^{4}$ called two-mode quadrature amplitudes, will be used to describe squeezing in a twophoton device in the SP:

$$
\begin{aligned}
\alpha_{1}(t)= & \left(\frac{\Omega+\epsilon}{2 \Omega}\right)^{1 / 2} a_{+} e^{i \Omega t} \\
& +\left(\frac{\Omega-\epsilon}{2 \Omega}\right)^{1 / 2} a_{-}^{\dagger} e^{-i \Omega t}, \\
\alpha_{2}(t)= & -i\left(\frac{\Omega+\epsilon}{2 \Omega}\right)^{1 / 2} a_{+} e^{i \Omega t} \\
& +i\left(\frac{\Omega-\epsilon}{2 \Omega}\right)^{1 / 2} a_{-}^{\dagger} e^{-i \Omega t} .
\end{aligned}
$$

The commutator algebra of these amplitudes is

$$
\begin{aligned}
& {\left[\alpha_{1}, \alpha_{1}^{\dagger}\right]=\left[\alpha_{2}, \alpha_{2}^{\dagger}\right]=\epsilon / \Omega,} \\
& {\left[\alpha_{1}, \alpha_{2}\right]=0,} \\
& {\left[\alpha_{1}, \alpha_{2}^{\dagger}\right]=\left[\alpha_{1}^{\dagger}, \alpha_{2}\right]=i ;}
\end{aligned}
$$

moreover, we will work in the modulation picture (MP), which is an interaction picture where the free time dependence at the carrier frequency $\Omega$ is transferred from the states to the operators (the states retaining the remaining free time dependence at modulation frequency $\epsilon$ ); the free Hamiltonian for the two output modes [Eq. (2)] is in the MP:

$$
H_{0}=H_{M},
$$

and the interaction Hamiltonian [Eq. (3)]:

$$
H_{I}=\frac{1}{2} \hbar i \kappa\left(a_{+} a_{-} e^{-2 i \varphi}-a_{+}^{\dagger} a_{-}^{\dagger} e^{2 i \varphi}\right) .
$$

In terms of the two-mode quadrature-phase amplitudes 
the total Hamiltonian, including the nonlinear dispersion term, has the form

$$
\begin{gathered}
H_{0}+H_{D}=\hbar \frac{1}{\lambda_{+}^{2} \lambda_{-}^{2}}\left[\left(\epsilon-\sigma \kappa^{\prime}\right)\left(i \alpha_{1}^{\dagger} \alpha_{2}-i \alpha_{2}^{\dagger} \alpha_{1}\right)\right. \\
\left.+\left(\kappa^{\prime}-\sigma \epsilon\right)\left(\alpha_{1}^{\dagger} \alpha_{1}+\alpha_{2}^{\dagger} \alpha_{2}\right)\right], \\
H_{I}=i \hbar \frac{k}{2} e^{-2 i \varphi}\left(\alpha_{1}^{+}+i \alpha_{2}^{\dagger}\right)\left(\alpha_{1}+i \alpha_{2}\right) \\
-i \hbar \frac{k}{2} e^{2 i \varphi}\left(\alpha_{1}^{\dagger}-i \alpha_{2}^{\dagger}\right)\left(\alpha_{1}-i \alpha_{2}\right)
\end{gathered}
$$

where $\lambda_{ \pm}=[(\Omega \pm \epsilon) / \Omega]^{1 / 2}, \sigma=\epsilon / \Omega$, and $k=\frac{1}{2} \kappa / \lambda_{+} \lambda_{-}$.

We will include damping in the nonlinear material by means of the usual term in the Hamiltonian, ${ }^{5}$ and the master equation for the reduced density matrix can be written

$$
\begin{aligned}
\frac{\partial \rho}{\partial t}= & A\left[\alpha_{1}^{\dagger} \alpha_{1}, \rho\right]+B\left[\alpha_{2}^{\dagger} \alpha_{2}, \rho\right]+C\left[\alpha_{2}^{\dagger} \alpha_{1}, \rho\right] \\
& +D\left[\alpha_{1}^{\dagger} \alpha_{2}, \rho\right]+\left(\frac{\partial \rho}{\partial t}\right)_{d},
\end{aligned}
$$

where

$$
\begin{aligned}
& A=i \frac{1}{\left(\lambda_{+} \lambda_{-}\right)^{2}}\left(\sigma \epsilon-\kappa^{\prime}\right)-\frac{i}{2} \frac{\kappa}{\lambda_{+} \lambda_{-}} \sin (2 \varphi), \\
& B=i \frac{1}{\left(\lambda_{+} \lambda_{-}\right)^{2}}\left(\sigma \epsilon-\kappa^{\prime}\right)+\frac{i}{2} \frac{\kappa}{\lambda_{+} \lambda_{-}} \sin (2 \varphi), \\
& C=\frac{1}{\left(\lambda_{+} \lambda_{-}\right)^{2}}\left(\sigma \kappa^{\prime}-\epsilon\right)+\frac{i}{2} \frac{\kappa}{\lambda_{+} \lambda_{-}} \cos (2 \varphi), \\
& D=\frac{1}{\left(\lambda_{+} \lambda_{-}\right)^{2}}\left(\epsilon-\sigma \kappa^{\prime}\right)+\frac{i}{2} \frac{\kappa}{\lambda_{+} \lambda_{-}} \cos (2 \varphi),
\end{aligned}
$$

and the damping term of the master equation reads

$$
\begin{aligned}
\left(\frac{\partial \rho}{\partial t}\right)_{d}=\gamma_{d} & \left(2 a_{+} \rho a_{+}^{\dagger}-a_{+}^{\dagger} a_{+} \rho-\rho a_{+}^{\dagger} a_{+}\right. \\
& \left.+2 a_{-} \rho a_{-}^{+}-a_{-}^{\dagger} a_{-} \rho-\rho a_{-}^{\dagger} a_{-}\right),
\end{aligned}
$$

where $\gamma_{d}$ is the damping constant.

\section{GENERALIZED FOKKER-PLANCK EQUATION}

The two-mode quasiprobability distribution (QPD) introduced by Schumaker and Caves $^{6}$ will be used here. Such distributions correspond to a $j$-ordered two-mode displacement operator for the two-mode quadraturephase amplitudes $\alpha_{m}$, which orders the two modes in the single-mode sense $\left(\alpha_{1}^{\dagger}\right.$ and $\alpha_{2}^{\dagger}$ always appear to the right of $\alpha_{1}$ and $\alpha_{2}$ in the case of antinormal ordering). Writing Eq. (11) in terms of the two-mode quadrature-phase amplitudes in the modulation picture and performing antinormal ordering in the former sense in Eqs. (10) and (11), we obtain the following Fokker-Planck equation for the QPD $P\left(\xi_{1}, \xi_{2}\right)$ :

$$
\begin{aligned}
\frac{\partial P}{\partial t}= & \left(\frac{\partial}{\partial \xi_{1}}\left(V_{1} \xi_{1}+W_{2} \xi_{2}\right) P+\frac{\partial}{\partial \xi_{2}}\left(V_{2} \xi_{1}+W_{2} \xi_{2}\right) P+c . c .\right) \\
& +\sum_{i, j=1,2} D_{i j} \frac{\partial^{2} P}{\partial \xi_{i} \partial \xi_{j}^{*}}
\end{aligned}
$$

where

$$
\begin{aligned}
& V_{1}=\gamma_{d}+i \epsilon+i \sigma k \sin (2 \varphi)+k \cos (2 \varphi), \\
& V_{2}=\kappa^{\prime}+k \sin (2 \varphi)-i \sigma k \cos (2 \varphi), \\
& W_{1}=-\kappa^{\prime}+k \sin (2 \varphi)-i \sigma k \cos 2 \varphi, \\
& W_{2}=\gamma_{d}+i \epsilon-i \sigma k \sin (2 \varphi)-k \cos (2 \varphi),
\end{aligned}
$$

and the diffusion matrix

$$
D=\gamma_{d}(1-\sigma)\left(\begin{array}{cc}
1 & -i \\
i & 1
\end{array}\right)
$$

It should be noted that this diffusion matrix is independent of the nonlinear coupling and has non-negative eigenvalues. This fact is a property of the employed $j$ ordered two-mode displacement operator. ${ }^{6}$

Since we will be interested in the symmetrized products of the two-mode quadrature-phase amplitudes, it is convenient to express Eq. (12) in terms of a QPD corresponding to operators written in symmetric order (Wigner QPD). ${ }^{6}$ It has been shown ${ }^{7}$ that for symmetric ordering the Fokker-Planck equation has the same drift matrix as for antinormal ordering [Eq. (12)] and the diffusion matrix is

$$
D=\gamma_{d}\left(\begin{array}{cc}
1+\sigma & -i(1-\sigma) \\
i(1-\sigma) & 1+\sigma
\end{array}\right)
$$

In order to study the noise properties of the output light we have calculated the covariance matrix $C_{m m}=\left\langle\left\langle\xi_{m} \xi_{n}^{*}\right\rangle\right\rangle=\left\langle\xi_{m} \xi_{n}^{*}\right\rangle-\left\langle\xi_{m}\right\rangle\left\langle\xi_{n}^{*}\right\rangle, m, n=1,2$, by means of standard methods. ${ }^{8}$ For simplicity, a unitary transformation $\xi=U \xi^{\prime}$ has been performed, that diagonalizes the diffusion matrix:

$$
U=\frac{1}{\sqrt{2}}\left(\begin{array}{ll}
i & 1 \\
1 & i
\end{array}\right)
$$

The average values in the new variables verify $\left\langle\xi^{\prime}\right\rangle_{t}=Y^{\prime}(t)\left\langle\xi^{\prime}\right\rangle_{0}$, where $Y^{\prime}(t)$ is the evolution matrix determined by the equation $\dot{Y}(t)=A^{\prime} Y^{\prime}(t), Y^{\prime}(0)=1$. The transformed drift matrix is

$$
A^{\prime}=-\left[\begin{array}{cc}
\gamma_{d}+i\left(\epsilon+\kappa^{\prime}\right) & -i k(1+\sigma) e^{2 i_{\varphi}} \\
i k(1-\sigma) e^{-2 i_{\varphi}} & \gamma_{d}+i\left(\epsilon-\kappa^{\prime}\right)
\end{array}\right] \text {, }
$$

and after some algebra it is obtained that the matrix elements $y_{m n}^{\prime}(t) \exp \left[-\left(\gamma_{d}+i \epsilon\right)\right]$ are 


$$
\begin{aligned}
& y_{11}^{\prime}(t)=\cosh \left(\frac{\kappa_{d} t}{2}\right)-2 i \frac{\kappa^{\prime}}{\kappa_{d}} \sinh \left(\frac{\kappa_{d} t}{2}\right), \\
& y_{12}^{\prime}(t)=i \frac{\kappa}{\kappa_{d}}\left(\frac{1+\sigma}{1-\sigma}\right)^{1 / 2} e^{2 i \varphi} \sinh \left(\frac{\kappa_{d} t}{2}\right), \\
& y_{21}^{\prime}(t)=-i \frac{\kappa}{\kappa_{d}}\left[\frac{1-\sigma}{1+\sigma}\right)^{1 / 2} e^{-2 i \varphi} \sinh \left(\frac{\kappa_{d} t}{2}\right), \\
& y_{22}^{\prime}(t)=\cosh \left(\frac{k_{d} t}{2}\right)+2 i \frac{\kappa^{\prime}}{\kappa_{d}} \sinh \left(\frac{\kappa_{d} t}{2}\right),
\end{aligned}
$$

where $\kappa_{d}=\left(\kappa^{2}-4 \kappa^{\prime 2}\right)^{1 / 2}$. It should be mentioned that if $\kappa^{\prime}=0$, we recover the evolution matrix without nonlinear dispersion. $^{3}$

On the other hand, it can be easily shown ${ }^{8}$ that the covariance matrix satisfies

$$
\begin{aligned}
C^{\prime}(t)= & Y^{\prime}(t) C^{\prime}(0) Y^{\prime^{\dagger}}(t) \\
& +\int_{0}^{t} Y^{\prime}(t) Y^{\prime-1}\left(t^{\prime}\right) D^{\prime}\left({Y^{\prime \dagger}}^{\dagger}\right)^{-1}\left(t^{\prime}\right) Y^{\prime \dagger}(t) d t^{\prime}
\end{aligned}
$$

The results for the covariance matrix and its transformed $C(t)=U C^{\prime}(t) U^{\dagger}$ are given in the Appendix. If we assume that the initial state is a two-mode coherent state, it is obtained as follows:

$$
\begin{aligned}
& \left(Y(t) C(0) Y^{\dagger}(t)\right)_{11}=e^{-2 \gamma_{d} t}\left[\frac{1}{4}\left[1-\frac{\kappa^{2}}{\kappa_{d}^{2}}-\frac{4 \kappa^{\prime 2}}{\kappa_{d}^{2}}+\frac{4 \kappa \kappa^{\prime} \sin \left[2 \varphi\left(1-\sigma^{2}\right)^{1 / 2}\right]}{\kappa_{d}^{2}}\right]\right. \\
& +\frac{1}{4}\left[1+\frac{\kappa^{2}}{\kappa_{d}^{2}}+\frac{4 \kappa^{\prime 2}}{\kappa_{d}^{2}}-\frac{4 \kappa \kappa^{\prime} \sin \left[2 \varphi\left(1-\sigma^{2}\right)^{1 / 2}\right]}{\kappa_{d}^{2}}\right] \cosh \left(\kappa_{d} t\right) \\
& \left.-\frac{1}{2} \frac{\kappa}{\kappa_{d}} \cos \left[2 \varphi\left(1-\sigma^{2}\right)^{1 / 2}\right] \sinh \left(\kappa_{d} t\right)\right], \\
& \left(Y(t) C(0) Y^{\dagger}(t)\right)_{22}=e^{-2 \gamma_{d} t}\left[\frac{1}{4}\left[1-\frac{\kappa^{2}}{\kappa_{d}^{2}}-\frac{4 \kappa^{\prime 2}}{\kappa_{d}^{2}}-\frac{4 \kappa \kappa^{\prime} \sin \left[2 \varphi\left(1-\sigma^{2}\right)^{1 / 2}\right]}{\kappa_{d}^{2}}\right]\right. \\
& +\frac{1}{4}\left[1+\frac{\kappa^{2}}{\kappa_{d}^{2}}+\frac{4 \kappa^{\prime 2}}{\kappa_{d}^{2}}+4 \frac{\kappa \kappa^{\prime} \sin \left[2 \varphi\left(1-\sigma^{2}\right)^{1 / 2}\right]}{\kappa_{d}^{2}}\right] \cosh \left(\kappa_{d} t\right) \\
& \left.+\frac{1}{2} \frac{\kappa}{\kappa_{d}} \cos \left[2 \varphi\left(1-\sigma^{2}\right)^{1 / 2}\right] \sinh \left(\kappa_{d} t\right)\right] \text {. }
\end{aligned}
$$

\section{NOISE IN THE QUADRATURE-PHASE AMPLITUDES}

It has been shown ${ }^{4}$ that the noise in the quadrature phases is given by

$$
\left\langle\left[\Delta E_{m}(\kappa, t)\right]^{2}\right\rangle=2 \Omega\left\langle\left|\Delta \alpha_{m}\right|^{2}\right\rangle, \quad m=1,2
$$

where $\left\langle\left|\Delta \alpha_{m}\right|^{2}\right\rangle$ is the symmetrically order squares variance of the two-mode quadrature-phase amplitudes:

$$
\left\langle\left|\Delta \alpha_{m}\right|^{2}\right\rangle=\frac{1}{2}\left\langle\alpha_{m} \alpha_{m}^{\dagger}+\alpha_{m}^{\dagger} \alpha_{m}\right\rangle-\left|\left\langle\alpha_{m}\right\rangle\right|^{2}, \quad m=1,2 .
$$

Thus, we are interested in the "reduced" spectral-density matrix:

$$
\Sigma_{m n}=\left\langle\Delta \alpha_{m} \Delta \alpha_{n}^{\dagger}\right\rangle_{\text {sym }}=\operatorname{tr}\left[\rho\left(\Delta \alpha_{m} \Delta \alpha_{n}^{\dagger}\right)_{\text {sym }}\right]=\left\langle\alpha_{m} \alpha_{n}^{\dagger}\right\rangle_{\text {sym }}-\left\langle\alpha_{m}\right\rangle\left\langle\alpha_{n}^{\dagger}\right\rangle,
$$

and, in particular, in its diagonal elements $\Sigma_{m m}=\left\langle\left|\Delta \alpha_{m}\right|^{2}\right\rangle$. It has been also shown ${ }^{4}$ that the uncertainties in $\alpha_{1}$ and $\alpha_{2}$ verify the uncertainty principle:

$$
\left\langle\left|\Delta \alpha_{1}\right|^{2}\right\rangle^{1 / 2}\left\langle\left|\Delta \alpha_{2}\right|^{2}\right\rangle^{1 / 2} \geq \frac{1}{2}\left|\left\langle\left[\alpha_{1}, \alpha_{2}^{\dagger}\right]\right\rangle\right|=\frac{1}{2} \text {. }
$$

Equation (22) describes squeezing since noise can be reduced below the zero-point level only by squeezing noise from one quadrature phase into the other. Moreover, the only states that yield equality in Eq. (22) are two-mode coherent states.

For an initial two-mode coherent state, and making use of the correspondence between density operators and quasiprobability distributions ${ }^{6}$ for symmetric ordering, the uncertainties in the two-mode quadrature-phase amplitudes are obtained from (20): 


$$
\begin{aligned}
& \Sigma_{11}=\left\langle\left|\Delta \alpha_{1}\right|^{2}\right\rangle=e^{-2 \gamma_{d} t}\left[\frac{2-\sin \left[2 \varphi\left(1-\sigma^{2}\right)^{1 / 2}\right]}{3}+\left\{-0.5+\sin \left[2 \varphi\left(1-\sigma^{2}\right)^{1 / 2}\right]\right\}^{-1} 3 \cos (\sqrt{3} \kappa t)\right. \\
& \left.-\frac{1}{2 \sqrt{3}} \cos \left[2 \varphi\left(1-\sigma^{2}\right)^{1 / 2}\right] \sin (\sqrt{3} \kappa t)\right)+\left(1-e^{-2 \gamma_{d} t}\right)\left(\frac{\left[2 \sigma-\left(1-\sigma^{2}\right)^{1 / 2}\right] \sin (2 \varphi)}{3}\right)-\frac{\gamma_{d}}{3 \kappa^{2}+4 \gamma_{d}^{2}} \\
& \times\left\{\frac{2 \gamma_{d}\left(\sigma-2\left(1-\sigma^{2}\right)^{1 / 2} \sin (2 \varphi)\right.}{3}+\kappa\left(1-\sigma^{2}\right)^{1 / 2} \cos (2 \varphi)+e^{-2 \gamma_{d} t}\right. \\
& \times\left[(\cos \sqrt{3} \kappa t)\left[\frac{2 \gamma_{d}\left[-\sigma+2\left(1-\sigma^{2}\right)^{1 / 2} \sin (2 \varphi)\right]}{3}-\kappa\left(1-\sigma^{2}\right)^{1 / 2} \cos (2 \varphi)\right]\right. \\
& \left.\left.+\left(\frac{\sin \sqrt{3} \kappa t}{\sqrt{3}}\right)\left[\kappa \sigma-2 \kappa\left(1-\sigma^{2}\right)^{1 / 2} \sin (2 \varphi)-2 \gamma_{d}\left(1-\sigma^{2}\right)^{1 / 2} \cos (2 \varphi)\right]\right]\right\}, \\
& \Sigma_{22}=\left\langle\left|\Delta \alpha_{2}\right|^{2}\right\rangle=e^{-2 \gamma_{d} t}\left(\frac{2+\sin \left[2 \varphi\left(1-\sigma^{2}\right)^{1 / 2}\right]}{3}-\frac{0.5+\sin (2 \varphi)\left(1-\sigma^{2}\right)^{1 / 2}}{3} \cos (\sqrt{3} \kappa t)\right.
\end{aligned}
$$$$
\left.+\frac{\cos \left[2 \varphi\left(1-\sigma^{2}\right)^{1 / 2}\right]}{2 \sqrt{3}} \sin (\sqrt{3} \kappa t)\right]+\left(1-e^{-2 \gamma_{d} t}\right) \frac{2 \sigma+\left(1-\sigma^{2}\right)^{1 / 2} \sin (2 \varphi)}{3}
$$$$
-\frac{\gamma_{d}}{3 \kappa^{2}+4 \gamma_{d}^{2}}\left\{\frac{2 \gamma_{d}\left[\sigma+2(1-\sigma)^{1 / 2} \sin (2 \varphi)\right]}{3}-\kappa\left(1-\sigma^{2}\right)^{1 / 2} \cos (2 \varphi)\right.
$$$$
+e^{-2 \gamma_{d} t}\left[[\cos (\sqrt{3} \kappa t)]\left[\kappa\left(1-\sigma^{2}\right)^{1 / 2} \cos (2 \varphi)-\frac{2 \gamma_{d}\left[\sigma+2\left(1-\sigma^{2}\right)^{1 / 2} \sin (2 \varphi)\right]}{3}\right]\right.
$$$$
\left.\left.+\left[\frac{\sin \sqrt{3} \kappa t}{\sqrt{3}}\right]\left[\kappa \sigma+2 \kappa\left(1-\sigma^{2}\right)^{1 / 2} \sin (2 \varphi)+2 \gamma_{d}\left(1-\sigma^{2}\right) \cos (2 \varphi)\right]\right]\right\},
$$

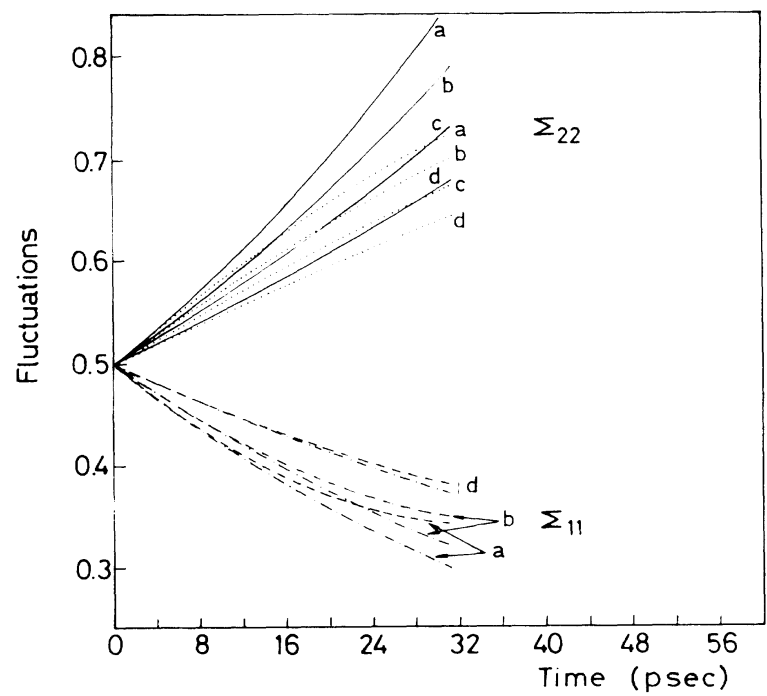

FIG. 1. Evolution of the uncertainties in the two-mode quadrature-phase amplitudes $\Sigma_{11}, \Sigma_{22}$ for $\kappa=1.75$, curve $a ; 1.50$, curve $b ; 1.25$, curve $c$; and 1.0 , curve, $d$. The normalized values of the other parameters were $\gamma_{d}=5 \times 10^{-4}, \sigma=0.28$, and $\varphi=0$. Curves labeled $(--$,$) and (\ldots)$ correspond to fluctuations with nonlinear dispersion.

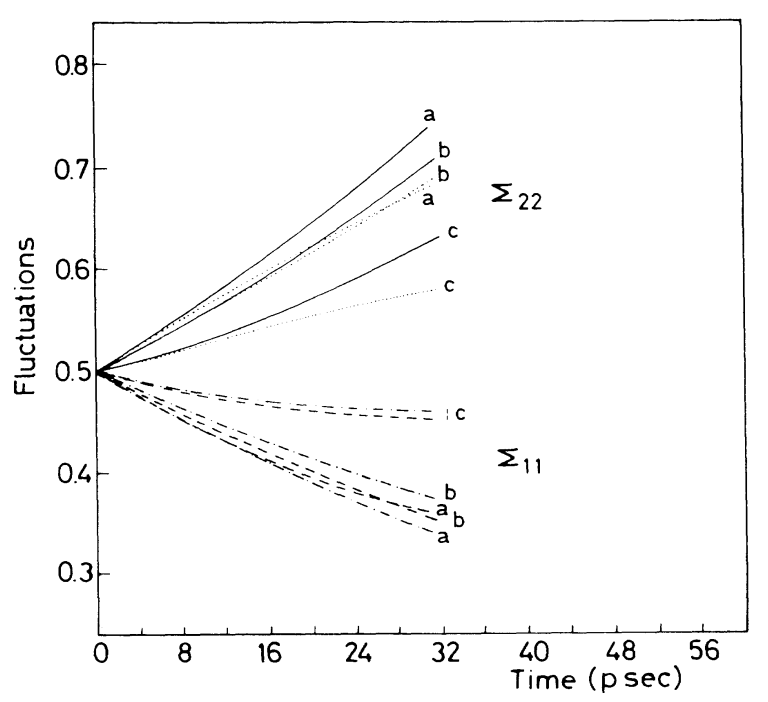

FIG. 2. Dependence of the fluctuations on the modulation $\sigma=\epsilon / \Omega$. Curve $a, \sigma=0.1$; curve $b, \sigma=0.6$; and curve $c$, $\sigma=0.9$. 


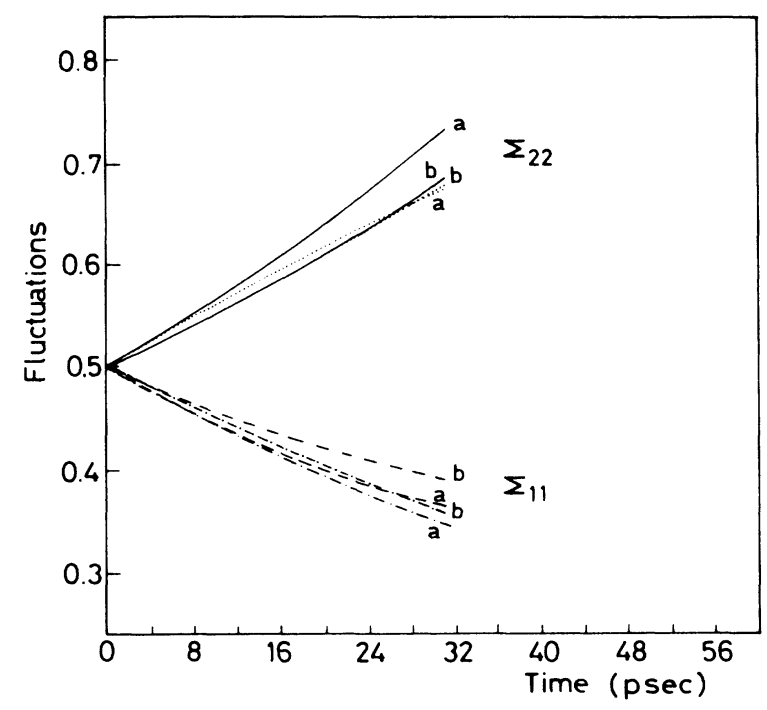

FIG. 3. Dependence of the fluctuations on the input phase $\varphi$. Curve $a, \varphi=0$; curve $b, \varphi=20^{\circ}$.

where we have made $\kappa^{\prime}=\kappa$. The uncertainties when nonlinear dispersion is not considered $\left(\kappa^{\prime}=0\right)$ are given in the Appendix.

We have studied the evolution of the noise in the quadrature-phase amplitudes along the nonlinear material for different values of the nonlinear coupling $\kappa$, the input phase $\varphi$, the modulation $\sigma=\epsilon / \Omega$, and the damping constant. Special attention has been paid to the limiting cases that can be studied within the model, i.e., high modulations $(\sigma \rightarrow 1)$, and high losses; moreover, the noises with and without nonlinear dispersion have been compared. Usual data $^{9}$ in squeezing experiments by parametric amplification have been employed in Figs. $1-4$, where the parameters are given in normalized values. It should be noted that the usual squeezing parameter ${ }^{10} s$ is $\kappa t / 2$ in our description.

The deleterious effect of nonlinear dispersion in the degree of squeezing attained can be observed in Fig. 1, where curves labeled $(-\cdot-\cdot-\cdot)$ are the fluctuations $\Sigma_{11}$ when nonlinear dispersion is neglected. On the other hand, the increase of the squeezing degree with the nonlinear coupling is also shown. The dependence of the noises in both quadrature-phase amplitudes $\Sigma_{11}$ and $\Sigma_{22}$ with the modulation is given in Fig. 2. It is worth noting that curves $a$ and $b$ correspond to $\sigma=0.1$ and 0.6 , respectively, while curve $c$ corresponds to $\sigma=0.9$; it is found that deleterious effect of high modulations on squeezing increases strongly from $\sigma=0.6$.

The behavior of the reduced fluctuations amplitude strongly depends on the input phase when nonlinear dispersion is considered. We have found that for $\varphi=10^{\circ}$, $\Sigma_{11}$ attains a minimum which depends on the nonlinear coupling $\kappa$, while the minimum is achieved at $\varphi=0$ when nonlinear dispersion is neglected. This behavior is in agreement with the results of Refs. 1 and 2. On the other

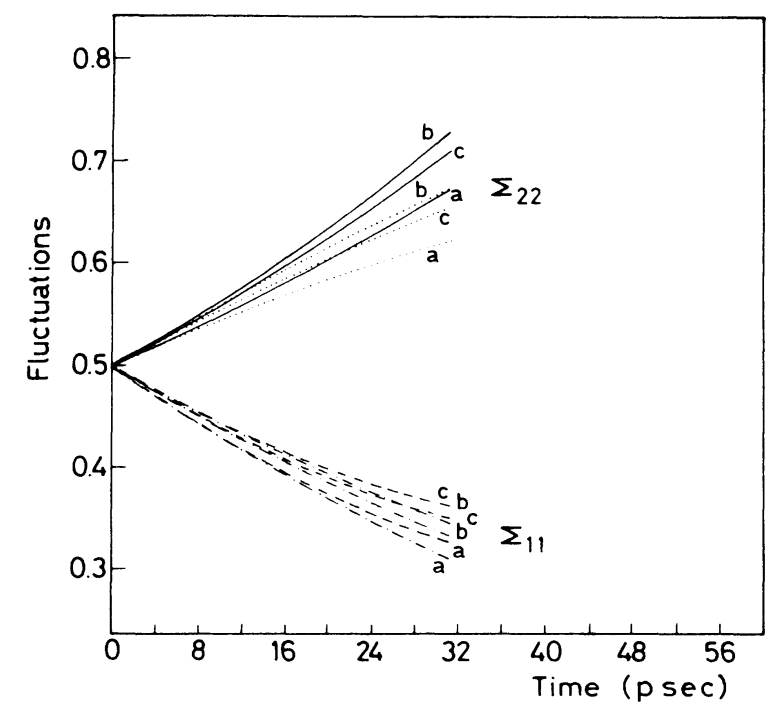

FIG. 4. Dependence of the fluctuations on the absorption losses; curve $a, \gamma_{d}=0.05$; curve $b, \gamma_{d}=0.2$; curve $c, \gamma_{d}=0.3$.

hand, we have also studied the dependence of the fluctuations on the damping constant and it has been observed that the deleterious effect of the absorption losses strongly increases from a certain value of $\gamma_{d}$ depending on the nonlinear coupling, while for smaller values of the damping constant, the squeezing degree remains practically constant; for example, for a value of the nonlinear coupling $\kappa=1.25$, this limit value for the absorption losses is $\gamma_{d}=0.1$ (Fig. 4).

In summary, we have analyzed the deleterious effects of nonlinear dispersion on the squeezing process in twophoton devices, taking into account the dependence of the fluctuations on the parameters which govern the process. It should be mentioned that the model employed enables us to study the process when the damping in the two modes $\Omega \pm \epsilon$ is different, ${ }^{11}$ such study being in progress at the present time. It should also be mentioned that this method is mainly suited to study nondegenerate parametric amplifiers, since it provides a direct dependence on the modulation frequency.

\section{ACKNOWLEDGMENT}

We would like to thank Professor R. Loudon for several useful discussions.

\section{APPENDIX}

The result of the second term of Eq. (17) for the covariance matrix

$$
I^{\prime}(t)=\int_{0}^{t} Y^{\prime}(t) Y^{\prime-1}\left(t^{\prime}\right) D^{\prime}\left({Y^{\prime}}^{\dagger}\right)^{-1}\left(t^{\prime}\right) Y^{\prime \dagger}(t) d t^{\prime}
$$

is 


$$
\begin{aligned}
& I_{11}^{\prime}(t)=(1+\sigma)\left[\frac{\kappa_{d}^{2}-\kappa^{2}}{2 \kappa_{d}^{2}}\left(1-e^{-2 \gamma_{d} t}\right)+\frac{\gamma_{d}}{\kappa_{d}^{2}-4 \gamma_{d}^{2}} \frac{\kappa^{2}}{\kappa_{d}^{2}}\left\{-2 \gamma_{d}+e^{-2 \gamma_{d} t}\left[\kappa_{d} \sinh \left(\kappa_{d} t\right)+2 \gamma_{d} \cosh \left(\kappa_{d} t\right)\right]\right\}\right], \\
& I_{12}^{\prime}(t)=I_{21}^{\prime *}=\left(1-\sigma^{2}\right)^{1 / 2} e^{2 i \varphi} \kappa\left(\frac{\kappa^{\prime}}{\kappa_{d}^{2}}\left(e^{-2 \gamma_{d} t}-1\right)\right. \\
& +\frac{\gamma_{d}}{\kappa_{d}^{2}-4 \gamma_{d}^{2}}\left\{-\frac{4 \kappa^{\prime} \gamma_{d}}{\kappa_{d}^{2}}-i+e^{-2 \gamma_{d} t}\left[\left(\frac{4 \kappa^{\prime} \gamma_{d}}{\kappa_{d}^{2}}+i\right) \cosh \left(\kappa_{d} t\right)\right.\right. \\
& \left.\left.\left.+\frac{2\left(\kappa^{\prime}+i \gamma_{d}\right)}{\kappa_{d}} \sinh \left(\kappa_{d} t\right)\right]\right\}\right) \\
& I_{22}^{\prime}(t)=-(1-\sigma)\left(\frac{\kappa_{d}^{2}-\kappa^{2}}{2 \kappa_{d}^{2}}\left(1-e^{-2 \gamma_{d} t}\right)+\frac{\gamma_{d}}{\kappa_{d}^{2}-4 \gamma_{d}^{2}} \frac{\kappa^{2}}{\kappa_{d}^{2}}\left\{-2 \gamma_{d}+e^{-2 \gamma_{d} t}\left[\kappa_{d} \sinh \left(\kappa_{d} t\right)+2 \gamma_{d} \cosh \left(\kappa_{d} t\right)\right]\right\}\right) .
\end{aligned}
$$

where the asterisk denotes the complex conjugate.

Transforming back the $I^{\prime}$ matrix, $I=U I^{\prime} U^{\dagger}$, we obtain

$$
\begin{aligned}
& I_{11}(t)=\left(1-e^{-2 \gamma_{d^{t}} t}\right)\left[\frac{\sigma}{2}\left(1-\frac{\kappa^{2}}{\kappa_{d}^{2}}\right]+\frac{\kappa \kappa^{\prime}\left(1-\sigma^{2}\right)^{1 / 2} \sin (2 \varphi)}{\kappa_{d}^{2}}\right] \\
& +\frac{\gamma_{d}}{\kappa_{d}^{2}-4 \gamma_{d}^{2}}\left\{\frac{2 \kappa \kappa^{\prime}}{\kappa_{d}^{2}} 2 \gamma_{d}\left(1-\sigma^{2}\right)^{1 / 2} \sin (2 \varphi)+\kappa\left(1-\sigma^{2}\right)^{1 / 2} \cos (2 \varphi)-\frac{2 \gamma_{d} \kappa^{2} \sigma}{\kappa_{d}^{2}}\right. \\
& +e^{-2 \gamma_{d} t}\left[\left[\cosh \left(\kappa_{d} t\right)\right] \mid 2 \gamma_{d} \frac{\kappa^{2} \sigma}{\kappa_{d}^{2}}-\frac{2 \kappa \kappa^{\prime}}{\kappa_{d}^{2}} 2 \gamma_{d}\left(1-\sigma^{2}\right)^{1 / 2} \sin (2 \varphi)-\kappa\left(1-\sigma^{2}\right)^{1 / 2} \cos (2 \varphi)\right) \\
& \left.\left.+\left[\sinh \left(\kappa_{d} t\right)\right]\left(\frac{\kappa^{2} \sigma}{\kappa_{d}}-\frac{2 \kappa \kappa^{\prime}}{\kappa_{d}}\left(1-\sigma^{2}\right)^{1 / 2} \sin (2 \varphi)-2 \gamma_{d} \frac{\kappa}{\kappa_{d}}\left(1-\sigma^{2}\right)^{1 / 2} \cos (2 \varphi)\right)\right]\right\}, \\
& I_{12}(t)=I_{21}^{*}(t)=\frac{1}{\kappa_{d}^{2}}\left[\frac{i\left(\kappa_{d}^{2}-\kappa^{2}\right)}{2}-\left(1-\sigma^{2}\right)^{1 / 2} \kappa \kappa^{\prime} \cos (2 \varphi)\right]\left(1-e^{-2 \gamma_{d} t}\right) \\
& +\frac{\gamma_{d}}{\kappa_{d}^{2}-4 \gamma_{d}^{2}}\left[\frac{-2 i \kappa^{2} \gamma_{d}}{\kappa_{d}^{2}}+\kappa\left(1-\sigma^{2}\right)^{1 / 2}\left\{\frac{1}{\kappa_{d}^{2}}\left[-4 \kappa^{\prime} \gamma_{d} \cos (2 \varphi)+\sin (2 \varphi)\right]\right.\right. \\
& +e^{-2 \gamma_{d} t}\left[\left[\kappa\left(1-\sigma^{2}\right)^{1 / 2}\right]\left[\frac{4 \kappa^{\prime} \gamma_{d} \cos (2 \varphi)}{\kappa_{d}^{2}}-\sin (2 \varphi)\right]\right. \\
& \left.+\frac{2 i \kappa^{2} \gamma_{d}}{\kappa_{d}^{2}}\right] \cosh \left(\kappa_{d} t\right) \\
& \left.\left.+\frac{\kappa}{\kappa_{d}}\left\{2\left(1-\sigma^{2}\right)^{1 / 2}\left[\kappa^{\prime} \cos (2 \varphi)-\gamma_{d} \sin (2 \varphi)\right]+i \kappa\right\} \sinh \kappa_{d} t\right\}\right] \text {, }
\end{aligned}
$$$$
I_{22}(t)=\left(1-e^{-2 \gamma_{d} t}\right)\left[\frac{\sigma}{2}\left(1-\frac{\kappa^{2}}{\kappa_{d}^{2}}\right]-\frac{\kappa \kappa^{\prime}\left(1-\sigma^{2}\right)^{1 / 2} \sin (2 \varphi)}{\kappa_{d}^{2}}\right]
$$$$
+\frac{\gamma_{d}}{\kappa_{d}^{2}-4 \gamma_{d}^{2}}\left\{-\frac{2 \kappa \kappa^{\prime}}{\kappa_{d}^{2}} 2 \gamma_{d}\left(1-\sigma^{2}\right)^{1 / 2} \sin (2 \varphi)-\kappa\left(1-\sigma^{2}\right)^{1 / 2} \cos (2 \varphi)-2 \gamma_{d} \frac{\kappa^{2} \sigma}{\kappa_{d}^{2}}\right.
$$$$
+e^{-2 \gamma_{d} t}\left[\left[\cosh \left(\kappa_{d} t\right]\left[2 \gamma_{d} \frac{\kappa^{2} \sigma}{\kappa_{d}^{2}}+\frac{2 \kappa \kappa^{\prime} 2 \gamma_{d}}{\kappa_{d}^{2}}\left(1-\sigma^{2}\right)^{1 / 2} \sin (2 \varphi)+\kappa\left(1-\sigma^{2}\right)^{1 / 2} \cos (2 \varphi)\right]\right.\right.
$$$$
\left.\left.+\left[\sinh \left(\kappa_{d} t\right)\right]\left[\frac{\kappa^{2} \sigma}{\kappa_{d}}+\frac{2 \kappa \kappa^{\prime}}{\kappa_{d}}\left(1-\sigma^{2}\right)^{1 / 2} \sin (2 \varphi)+2 \gamma_{d} \frac{\kappa}{\kappa_{d}}\left(1-\sigma^{2}\right)^{1 / 2} \cos (2 \varphi)\right]\right)\right\} .
$$

When nonlinear dispersion is not considered, $\kappa^{\prime}=0$, we obtain from (18) and (25) the following uncertainties in the two-mode quadrature-phase amplitudes: 


$$
\begin{aligned}
& \Sigma_{11}=\left\langle\left|\Delta \alpha_{1}\right|^{2}\right\rangle=\frac{1}{2} e^{-2 \gamma_{d} t}\left[\cosh (\kappa t)-\left(1-\sigma^{2}\right)^{1 / 2} \cos (2 \varphi) \sinh (\kappa t)\right] \\
& +\frac{\gamma_{d}}{\kappa^{2}-4 \gamma_{d}^{2}}\left(\kappa\left(1-\sigma^{2}\right)^{1 / 2} \cos (2 \varphi)-2 \gamma_{d} \sigma\right. \\
& +e^{-2 \gamma_{d} t}\left\{[\cosh (\kappa t)]\left[2 \gamma_{d} \sigma-\kappa\left(1-\sigma^{2}\right)^{1 / 2} \cos (2 \varphi)\right]\right. \\
& \left.\left.+[\sinh (\kappa t)]\left[\kappa \sigma-2 \gamma_{d}\left(1-\sigma^{2}\right)^{1 / 2} \cos (2 \varphi)\right]\right\}\right), \\
& \Sigma_{22}=\left\langle\left|\Delta \alpha_{2}\right|^{2}\right\rangle=\frac{1}{2} e^{-2 \gamma_{d} t}\left[\cosh (\kappa t)+\left(1-\sigma^{2}\right)^{1 / 2} \cos (2 \varphi) \sinh (\kappa t)\right. \\
& +\frac{\gamma_{d}}{\kappa^{2}-4 \gamma_{d}^{2}}\left(-\kappa\left(1-\sigma^{2}\right)^{1 / 2} \cos (2 \varphi)-2 \gamma_{d} \sigma\right. \\
& +e^{-2 \gamma_{d} t}\left\{[\cosh (\kappa t)]\left[2 \gamma_{d} \sigma+\kappa\left(1-\sigma^{2}\right)^{1 / 2} \cos (2 \varphi)\right]\right. \\
& \left.\left.+[\sinh (\kappa t)]\left[\kappa \sigma+2 \gamma_{d}\left(1-\sigma^{2}\right)^{1 / 2} \cos (2 \varphi)\right]\right\}\right) .
\end{aligned}
$$

${ }^{1}$ M. D. Levenson, R. M. Shelby, and S. H. Perlmutter, Opt. Lett. 10, 514 (1985).

${ }^{2}$ M. D. Levenson and R. M. Shelby, in Quantum Optics $I V$, edited by J. D. Harvey and D. F. Walls (Springer-Verlag, Berlin, 1986), p. 2.

${ }^{3}$ G. J. Milburn, M. D. Levenson, R. M. Shelby, S. H. Perlmutter, R. G. Devoe, and D. F. Walls, J. Opt. Soc. Am. B 4, 1476 (1987).

${ }^{4}$ C. M. Caves and B. L. Schumaker, Phys. Rev. A 31, 3068 (1985); L. Sainz, P. García Fernández, and F. J. Bermejo, Phys. Lett. A 130, 87 (1988).

$5 \mathrm{~J}$. Perina, in Quantum Statistics of Linear and Nonlinear Optical Phenomena (Kluwer Academic, Norwell, MA, 1984), Chaps. 7, 8, and 9 .
${ }^{6}$ B. L. Shumaker and C. M. Caves, Phys. Rep. 135, 317 (1986); S. M. Barnett and P. L. Knight, J. Mod. Opt. 34, 841 (1987); B. R. Mollow and R. J. Glauber, Phys. Rev. 160, 1076 (1987); G. J. Milburn, J. Phys. A 17, 737 (1984); K. E. Cahill and R. J. Glauber, Phys. Rev. 177, 1883 (1969).

${ }^{7}$ L. Sainz (private communication).

${ }^{8}$ N. G. Van Kampen, Stochastic Processes in Physics and Chemistry (North-Holland, Amsterdam, 1981), p. 228.

${ }^{9}$ L. A. Wu, M. Xiao, and H. J. Kimble, J. Opt. Soc. Am. B 4, 1465 (1987).

${ }^{10}$ M. J. Collett and R. Loudon, J. Opt. Soc. Am. B 4, 1525 (1987).

${ }^{11}$ A. S. Lane, M. D. Reid, and D. F. Walls, Phys. Rev. Lett. 60, 1940 (1988). 